\title{
Certificación de calidad en los archivos. Análisis y prospectiva
}

\author{
Manuela Moro Cabero*
}

Resumen: Análisis del alcance de la certificación en la práctica archivística. Descripción del proceso certificador, de la normativa específica utilizada para ello y de los organismos certificadores, con el fin de mostrar su funcionamiento y difundir fuentes y agentes que intervienen en la certificación de Archivos. Contextualización de la certificación en el modelo de gestión de documentos — serie ISO 30300—, sistema de gestión para los documentos, con el objetivo de difundir sus principios y estructura donde se regula su certificación. Valoración del impacto de la certificación sobre el mantenimiento de sistemas de gestión documental eficientes en las organizaciones. El método analítico-descriptivo es utilizado en el estudio, cuyos resultados son: descripción del(los) objeto(s) y proceso de certificación en el Archivo. Presentación del proyecto de certificación regulado en la serie de normas ISO 30300 sobre sistemas de gestión documental: objeto, proceso y agentes implicados. Reflexión sobre el impacto del proceso certificador de la actividad de archivar en las organizaciones.

Palabras claves: Certificación, gestión de documentos, ISO 15489:2001, serie ISO 30300.

\section{Certification in archival practice: analysis and outlook}

Abstract: An analysis of the reach of certification in archival practice. The article describes the certification process and the specific standards used in it, as well as the agencies responsible for certification. The purpose is to show how the process works and to make known the sources and agents involved in archival certification. Certification is contextualized within the records management model (ISO 30300: Management Systems for Records), in order to promote this model's principles and structure for regulating certification. There is an assessment of the impact of certification on maintaining efficient records management systems in organizations. The analyticaldescriptive method used in the study results in a description of the object(s) and archival certification process. This presentation of a project of certification of a records management system according to ISO 30300 - object, process and agents involved-concludes with a reflection on the impact of the certification process on organizations.

Key words: Certification, records management, ISO 15489:2001, SERIE ISO 30300.

*Universidad de Salamanca, Facultad de Traducción y Documentación. Correo-e:moroca@usal.es. Recibido: 08-11-2010; 2. ${ }^{\mathrm{a}}$ versión: 21-03-2011; aceptado: 23-03-2011. 


\section{Introducción}

La certificación en los Archivos, con mayúscula (Heredia, 2007), se circunscribe a la certificación del logro de la calidad, en sistemas y servicios, así como de personas. Mientras que la certificación de la calidad y el reconocimiento de la excelencia es práctica habitual en diferentes países, no lo es tanto la certificación de personas. La certificación de los sistemas de gestión de documentos no se ha llevado a cabo de modo expreso, siendo efectuadas evaluaciones relacionadas, únicamente, con objetivos de calidad o de excelencia. El interés manifestado por la comunidad archivística sobre la calidad y su certificación es paralelo al interés mostrado por las organizaciones, dado que el Archivo, inmerso en su estructura, es considerado un sistema o un servicio sobre el que demostrar su eficiencia. La literatura archivística dispone de numerosos referentes sobre la calidad provenientes de diferentes escuelas de pensamiento archivístico (Brumm, 1996; Moro Cabero,1997 y 2009; Roberge, 2002; Núñez Fernández, 2007: Duff y Mckemmish, 2000, etc.)

La Asociación Española de Normalización (AENOR, 2010) define la certificación como "la acción llevada a cabo por una entidad independiente de las partes interesadas mediante la que se manifiesta que una organización, producto, proceso o servicio, cumple los requisitos definidos en unas normas o especificaciones técnicas". Se trata de una acción en la que se implica a una entidad independiente y que es de carácter voluntario. El reconocimiento de la capacidad o dotación de autoridad de "esa entidad independiente" se denomina acreditación. La acreditación es un proceso de autorización, mediante el cual un organismo certificador (entidad independiente que testifica) se somete a evaluación y es realizado por un organismo de acreditación único para cada país. Tanto para la realización de las certificaciones como para las acreditaciones debe adoptarse un método normalizado.

En este estudio se pretenden contextualizar la práctica de certificación de la calidad en el Archivo. Para ello, se analizará el proceso certificador y la relación de normas sobre las que se regula, así como la naturaleza y competencia de los agentes que intervienen en el proceso. Se argumentará sobre la proyección de futuro de la certificación en sistemas de gestión documental. El debate se fundamenta en el análisis de los borradores de la serie ISO 30300 sobre sistemas normalizados de gestión documental. Finalmente se pretende aportar una reflexión sobre el valor que la certificación implica en el ejercicio de archivar y, más concretamente, en los organismos de producción del objeto archivado.

El método utilizado es analítico-descriptivo basado en la revisión de recursos bibliográficos, normativos y legislativos. Los resultados obtenidos son fruto de una sistematizada reflexión de carácter prospectivo.

El ensayo se estructura en un primer epígrafe en el que se contextualiza la actividad certificadora en la comunidad archivística para demostrar su alcance, naturaleza y práctica habitual. Destinamos un segundo enunciado al análisis detallado del proceso certificador y a la descripción de la normativa básica así como 
de los principales agentes. Conocido el contexto y comprendido el proceso certificador se aborda la proyección certificadora en los sistemas de gestión de documentos. Para ello se analiza el rol de la certificación en el nuevo modelo de gestión de documentos (ISO 30300). Finalmente, se presentan a modo de conclusión algunas reflexiones sobre el valor e impacto de la certificación en el Archivo.

\section{Calidad y contexto certificador en los archivos}

La simplificación de la estructura normativa sobre calidad, serie ISO 9000:2000, así como el surgimiento de los premios de reconocimiento de la excelencia en los años 90 son significativos para que las organizaciones muestren mayor interés por la calidad, incluidos los Archivos. Actualmente, muchos son los centros y sistemas de archivos que están inmersos en la implantación de sistemas de calidad o en la verificación de su excelencia mediante la adopción de modelos de excelencia. Calidad y excelencia forman parte habitual de los valores y la misión de buen número de organizaciones. Se trata de un modelo de gestión basado en un enfoque sistémico, de procesos y orientado hacia sus clientes: capital humano, clientes-usuarios, proveedores, terceras partes e interesados. Aunque la finalidad de estos modelos es la de alcanzar (o aproximarse a) la calidad total en procesos de producción o de servicio, en la administración de agentes así como en la calidad de vida laboral en la organización, el método para su consecución difiere: constatamos un modelo basado en normas (serie ISO 9000) y otro, de reconocimiento de la excelencia, centrado en la autoevaluación de la gestión estructurada en criterios y subcriterios mediante su cuantificación (Modelo de la Fundación Europea para la Gestión de la Calidad, EFQM).

El modelo basado en normas ISO exige sensibilización, formación, diseño, implementación, evaluación de un sistema de gestión de calidad. El éxito de su adopción radica en su fundamentación como sistema normalizado de gestión (MSS) estructurado en el dominio conceptual (ISO 9000, vocabulario y principios), el control de requisitos (ISO 9001), su implantación (ISO 9004) y su certificación (ISO 19011:2002; ISO 17021:2011). Este modelo ha sido seguido por numerosos archivos, aportamos algún ejemplo del Estado Español, como es el caso del Archivo de la Diputación de Alicante (desde el 2001), el Archivo Universitario de la Universidad Complutense (2008) o el Archivo Nacional de Cataluña (desde 2004).

El modelo basado en el reconocimiento de la excelencia difiere del anterior en estructura y alcance, ya que NO se basa en normas y se centra en la autoevaluación y la mejora continuada. Se fundamenta en la lógica reder: resultados (aquello que la organización consigue), enfoque (aquello que la organización piensa hacer y las razones para ello), despliegue (aquello que realiza la organización para poner en práctica el enfoque), evaluación y revisión (aquello que hace la organización para evaluar y revisar el enfoque y despliegue). El resultado cuan- 
tificado de la secuencia reder se materializa en un cuadro de indicadores de mando integral (liderazgo, políticas y estrategias, procesos, colaboradores y recursos, personas), que nos ayudan a conocer a través de los resultados obtenidos (en clientes, personal, sociedad y rendimiento final de la organización) si se está creando el futuro deseado. Su retroalimentación se activa mediante la innovación y el aprendizaje. Ejemplo de adopción en España de este modelo lo hallamos en el Sistema de Archivos de Castilla y León, cuya cabecera del sistema ha adaptado una herramienta de medición al contexto archivístico (2006) y mantiene un ejemplo de gestión con sello de calidad reconocido como es el caso del Archivo Histórico Provincial de León (AHPLe). Un segundo ejemplo lo representa el Archivo Universitario de Castilla La-Mancha, que en el 2004 obtuvo su primer reconocimiento EFQM.

Los citados modelos (EFQM-Normalizado) no son incompatibles. De hecho, son significativas las instituciones que adoptan un modelo basado en el reconocimiento de la excelencia y con posterioridad, implementan un modelo normalizado de certificación o viceversa. El AHPLe ha implementado un sistema de gestión basado en la norma UNE-EN-ISO 9001:2000 y ha recibido el reconocimiento EFQM de excelencia 300+. El primer modelo se fundamenta en el cumplimiento y verificación de la conformidad a las normas, el segundo, pretende demostrar el cumplimiento de los criterios contenidos en los modelos (EFQM o adaptaciones en el marco de las Administraciones públicas, tales como el modelo del Marco Común de Evaluación-CAF y el Modelo de evaluación, aprendizaje y mejora-EVAM). Los criterios (y sus subcriterios) componentes de estos prototipos conforman las divisiones (y subdivisones) en las que se analiza el modo de gestión. Estos últimos, permiten la comparación del logro en los servicios o unidades aplicadas, puesto que el conjunto de subcriterios es cuantificable mediante datos y ratios obtenidos de mediciones de percepciones, rendimiento y de indicadores clave o de resultados. La implementación de un modelo de reconocimiento de la excelencia, a nuestro entender, resulta de gran utilidad, para la adopción del modelo basado en normas.

Ambos modelos contribuyen a orientar el trabajo archivístico hacia un fin estratégico para las organizaciones; si bien, mientras que en uno han venido utilizándose, únicamente, conceptos de mención y de reconocimiento (EFQM y adaptaciones), en el otro se utiliza el concepto de certificación. Este hecho ha sido modificado recientemente, dado que es posible certificar el modelo EFQM y sus adaptaciones. De hecho, mediante resolución (2009) se regula su procedimiento.

La evaluación de la gestión de los servicios en los centros universitarios ha sido acometida mediante versión adaptada EFQM, siendo promovida por diferentes planes nacionales de evaluación de la calidad a través de la ANECA —Agencia Española de Evaluación de la Calidad- o en algunas CC.AA. por las agencias específicas competentes.

La práctica de certificación en los archivos españoles no sólo afecta a su modelo de gestión, sino que se expande a otros compromisos de calidad específicos 
como son los compromisos de servicio en los archivos de Administraciones Públicas. Dicho compromiso de calidad requiere la adopción de una metodología específica cuyo resultado es una herramienta denominada Carta de servicios, legislada mediante Real Decreto (España, 2005) y dotada de un método de elaboración (2006), en cuya guía se especifica el procedimiento, en su caso, de certificación.

En suma, los archivos han asumido modelos certificados de gestión de la calidad y excelencia en los sistemas de gestión y en los servicios prestados. De hecho, la atracción por la calidad es observable en las disposiciones que los sistemas de archivos recogen en sus planes de actuación, donde la calidad es un foco de interés y un elemento de proyección estratégica (Junta de Castilla y León, 2010). A este respecto, existiendo una práctica certificadora de sistemas, productos y servicios, es comprensible la propuesta certificadora del sistema de gestión para los documentos que incluye la nueva normativa ISO 30300, aspecto que analizaremos en el enunciado 4 , una vez comprendidos los procesos de acreditación y de certificación.

\section{El proceso de acreditación y de certificación}

\subsection{La acreditación de entidades de certificación}

Los conceptos de certificación y acreditación permiten entender la secuencia del proceso de autorización y responsabilidad en la certificación dado que, una entidad propone un sistema de gestión o producto-servicio a un organismo independiente para su certificación; Lógicamente, la autoridad debe ser regulada y para ello existen normas de certificación y de acreditación. Tres son los tipos de normas aplicadas de modo coordinado aunque empleadas con finalidades distintas: a) normas de acreditación; b) normas de certificación, y c) normas de evaluación o auditoría. Detallemos la secuencia para cada una de ellas.

a) Normas de acreditación

La norma ISO/IEC 17021:2011 regula los requisitos para los organismos que realizan la auditoría y la certificación de sistemas de gestión. En ella se establecen los requisitos para un adecuado funcionamiento de las entidades certificadoras de modo competente, coherente e imparcial, responsable, confidencial y transparente, permitiendo dar respuesta a posibles quejas. Siguiendo estos preceptos, en el contexto de la seguridad de la información ha sido editada una normativa de auditoría y certificación: ISO/IEC 27006:2007, aunque en la anterior norma se recogen pautas generales aplicables a cualquier sistema de gestión. Dos son las normas enunciadas, por tanto, que se encuentran activas y que son utilizadas por el organismo de acreditación único nombrado en cada país (España: ENAC-Entidad Nacional de Acreditación). 


\section{b) Normas de certificación}

Los organismos acreditados para realizar las auditorías de certificación, mediante equipos de auditores operan auditando el cumplimiento de determinadas normativas. Las entidades auditoras disponen de normas de evaluación y certificadoras. En el caso de los sistemas de calidad y medioambiente se ha utilizado la normativa ISO 19011:2002 en la que se regulan directrices para auditar los sistemas de gestión de calidad y de gestión medioambiental, incluyendo en las mismas pautas para el establecimiento del programa auditor, la implementación de dicho programa y su seguimiento y revisión. Directrices específicas sobre el programa y plan auditor han sido regulados en la norma ISO/IEC 17021:2011. De igual modo, para evaluar los sistemas de seguridad de la información se utiliza la normativa ISO/IEC 27006.2007, anteriormente citada, donde se recogen directrices con idéntico fin de facilitar y avalar el proceso auditor.

c) Normas de evaluación.

Los organismos que soliciten la certificación, deberán demostrar el cumplimiento de la normativa de requisitos del sistema de gestión (calidad, medioambiente, seguridad de la información, etc.). Una vez comprobado, serán certificados. Lógicamente, la normativa a verificar será la específica a los entornos de gestión que se pretenden certificar (calidad, ambiental, seguridad) y/o servicios (disposiciones de cumplimiento para carta de servicios).

Para finalizar, resumimos la secuencia del proceso de acreditación como sigue: una entidad única en cada país (AENOR, etc.), regulada su creación y funcionamiento en un marco legislativo, acredita organismos, tomando en consideración las directrices de la norma ISO/IEC 17021:2011 (en el caso de sistemas, productos y personas). Las entidades acreditadas tienen competencia para certificar la conformidad de normas. Estos organismos pueden certificar calidad, seguridad de la información, gestión ambiental, etc., y en estudio, gestión documental. A tal efecto, proceden tomando en consideración las directrices de la norma ISO 19011:2002, en las actuaciones de certificación de calidad y de gestión medioambiental, y de la norma ISO 27006:2007 para las actuaciones de seguridad de la información, otras directrices para los sistemas de gestión se incluyen igualmente en ISO/IEC 17021:2011. Se estudia la valoración de la norma ISO/IEC 17021 para su aplicación en la gestión documental. Los organismos certificadores evalúan la conformidad del cumplimiento de la normativa de calidad (serie ISO 9000), seguridad de la información (serie ISO 27000) y, en proyecto, la gestión documental (serie ISO 30300)

\subsection{El proceso certificador de la conformidad}

Una consulta rápida a la Web de cualquier entidad certificadora incluye las etapas del proceso certificador. En detalle, son numeradas como sigue: 1. Selección del organismo certificador; 2. Cumplimentado de documentos (formularios) 
de solicitud; 3. Revisión de la documentación del centro por el organismo certificador; 4. Visita previa (opcional; no todos los organismos la realizan) a la certificación; 5. Auditoría de certificación; 6. Presentación de acciones correctoras; 7. Concesión de certificado, y 8. Auditorías de seguimiento y de renovación.

De acuerdo con Santiago Palom (1998), dichas etapas pueden ser agrupadas en 3 fases significativas: fase de solicitud de la certificación, fase de auditoría y fase de certificación.

En la primera fase (etapas 1-3), se selecciona el organismo certificador, considerando criterios objetivos — datos operativos - y subjetivos —empatía, imagen, etc.- . Elegido el organismo certificador, es preciso cumplimentar la documentación de solicitud, en la que se especifican datos genéricos de la institución, persona de contacto, normativa por la que se certifica, actividades y productos cubiertos por el sistema, ubicaciones físicas del organismo, organigrama general y otros datos de interés para el proceso. En esta fase será revisada la documentación del centro por el organismo certificador quién informa al respecto señalando las contradicciones.

En la segunda fase (etapas 4 a 6), se designan los auditores o equipo auditor y se realiza (a petición) una visita previa o de contacto, en la que se revisa la documentación demostrativa de la implantación del sistema de gestión y de su funcionamiento. Los auditores en esta fase ejecutan la auditoría, propiamente dicha, destacando tres etapas:

a) Etapa de reunión inicial con la dirección del centro para intercambiar expectativas y guía de trabajo avanzando posibles resultados con objeto de evitar generación de falsas expectativas.

b) Etapa de obtención de evidencias para verificar el estado de implantación del sistema y negociar las resoluciones de algunas disconformidades. La tarea final, se centrará en redactar un informe previo que se utilizará como elemento de diálogo en la etapa final.

c) Etapa de reunión final donde se informa sobre la situación verificada. El informe será empleado como documento de referencia. Los auditores informarán sobre la situación aunque carecen de capacidad para otorgar la certificación. Esta petición será trasladada al Comité de Evaluación del Organismo Certificador, quien, a raíz del informe de los auditores, estimará su certificación.

En la tercera fase (etapas 7 y 8), si se cumplen los requisitos de evaluación, la entidad recibe la certificación que lógicamente, debe ser celebrada como un éxito para el Archivo y la organización. Finalmente, el organismo que concede la certificación debe asegurarse de que el sistema se mantiene conforme y, por tanto, suele realizar visitas a los centros certificados de seguimiento (anuales), de renovación (antes de los 3 años cumplidos de la concesión del certificado) y extraordinarias, ante hechos o eventos concretos como una denuncia expresa.

Finalmente, señalamos que las certificaciones de las cartas de servicios se enunciaban y regulaban en la parte II de la Guía de elaboración de cartas de 
servicios (2006, p.45 y ss.) promovida por la AEVAL- Agencia de Evaluación de la Calidad. Desde el 2009, su Consejo Rector ha reglamentado la certificación de las cartas de servicios de las organizaciones de la Administración Pública (2009). De hecho, en la página Web de la AEVAL (2010) se especifica el alcance de la certificación como sigue:

«El procedimiento de certificación no se realiza únicamente sobre el contenido de la Carta en sí, sino también sobre la metodología que se ha seguido para su elaboración y el trabajo desarrollado a tal efecto, sobre el cumplimiento de los compromisos de calidad y el seguimiento de los indicadores considerados en la misma y sobre el establecimiento de los criterios para su revisión periódica”.

\section{La certificación de sistemas de gestión documental}

La normalización de sistemas de gestión documental es de responsabilidad del Subcomité Técnico 11 sobre gestión de documentos y Archivos (SC11), integrante del Comité Técnico 46 de ISO, quien ha desarrollado el código de buenas prácticas ISO 15489 (2001). El éxito en la adopción del código animó al SC11, en el 2007, a la concreción de una nueva propuesta de reconocimiento de un sistema de gestión para los documentos (Bustelo; 2009). ISO ha aceptado dicho marco normativo de sistemas de gestión (MSS); cuyo alcance y estructura difiere, lógicamente, del código de buenas prácticas, como la serie denominada ISO 30300. Su estructura operativa se conforma por un conjunto de normas (estructura típica de un MSS) perfilándose un modelo normalizado de sistemas de gestión para los documentos. El contexto ISO MSS es específico de determinadas series de sistemas de seguridad, de calidad, de gestión medioambiental, de transportes, etc. De hecho, el Join Technical Coordination Group for MSS (JTCG) pretende coordinar, terminología, fundamentos, estructura y marco de acreditación y certificación de dichas normas.

El modelo normaliza el sistema de gestión y se comprende como el proveedor de un conjunto de procesos y de herramientas que permiten de modo verificable y sistemático el control organizacional en un entorno de producción basado en buenas prácticas de gestión. El modelo MSR (Management System for Records) es aplicable en cualquier organización con independencia del tamaño y modelo empresarial adoptado. Posibilita el establecimiento de políticas y de objetivos de gestión documental, definiendo funciones y distribuyendo responsabilidades; apuesta por la construcción de una arquitectura de procesos sistemáticos y la medición y evaluación de objetivos y rendimiento de los mismos; Asegura, además, la conformidad con los requisitos documentales, mediante la incorporación y mantenimiento de mecanismos de revisión y mejora continuada (ISO/DIS 30301:2010).

El éxito de este reconocimiento MSS está en consonancia con el grado de relaciones que mantiene con otros modelos MSS (requisitos documentales de sistemas normalizados de gestión de la calidad, gestión ambiental, gestión de se- 
guridad de la información, etc.) Las analogías y sinergias son cuantiosas, facilitando su empleo y comprensión. Entre las sinergias destacamos: el reconocimiento ISO y, por tanto, su proyección internacional; el carácter facultativo de su adopción como código de buenas prácticas; la estructura normativa similar (principios y vocabulario, requisitos, directrices de implantación, pautas de evaluación, acreditación y de certificación); el método de gestión; la importancia otorgada al análisis y gestión del riesgo; el enfoque a procesos, la orientación hacia la mejora continuada; la correlación de los requisitos de evidencia documental, etc. Entre sus sinergias destacamos el rol colaborador o de soporte mutuo que implica este sistema de gestión documental respecto al cumplimiento de los requisitos de verificación y de evidencia basados en un riguroso soporte documental. Un segundo foco sinérgico es su coordinación hacia una terminología, fundamentación, estructura y normativa común de acreditación y certificación, simplificando su comprensión y favoreciendo su empleo y asimilación. En suma, su particular reconocimiento como sistemas normalizados de gestión (MSS).

Bajo el título "Management system for records - MSR" el sistema normalizado de gestión documental se conforma como serie ISO 30300 con dos productos muy avanzados cuya edición se estima para el segundo semestre del 2011 (Bustelo y Ellis, 2010):

a) Una norma de principios y vocabulario, codificada como ISO 30300 (borrador FDIS). En ella se explica la concepción, los principios, la aplicación MSR así como la estructura de la serie ISO 30300 y su proyección.

b) Una norma de especificación de requisitos, codificada como ISO 30301 (borrador FDIS). En ella se detalla el contexto organizacional, directrices sobre liderazgo y requisitos. Se incluyen varios anexos significativos sobre: relaciones entre normas MSS, relación de procesos y controles, lista de parámetros de autoevaluación.

En la introducción de estos borradores se hace referencia a otros proyectos de norma. Estos son codificados como:

- ISO 30302. Guía de implantación del MSR.

- ISO 30304. Guía de autoevaluación del sistema.

- ISO 30303. Esta codificación estaría destinada a regular requisitos del procedimiento certificador y de acreditación en un proceso evaluador de tercera parte. En ella se detallarán los requisitos para la auditoría y certificación de sistemas de gestión documental, siempre y cuando no se estimen oportunas las directrices de las normas mencionadas con anterioridad ISO 19011:2002 e ISO/ IEC 17021:2011, tomando en consideración los objetivos de trabajo del JTCG.

Al MSR se vinculan a nivel operativo informes técnicos y normas orientadas a regular la gestión de metadatos, el proceso de digitalización, la migración, el análisis del trabajo, las normas ISO 15489:2001, etc. 


\section{Análisis del valor e impacto de la certificación en el archivo}

El propósito de un sistema de gestión de documentos se detalla en el borrador de la norma ISO/DIS 30300 (2010) bajo el objetivo de crear y controlar los documentos de modo sistemático y verificable, de tal forma que permita el cumplimiento de requisitos de carácter legislativo y normativo (conformidad marco legislativo, procedimental y normativo), operativo (continuidad de negocio, gestión de riesgos, prevención de desastres, organización del trabajo, etc.) y cultural, referido al logro de las expectativas de usuarios y de la corporación. Beneficios del modelo vinculados al propósito son subrayados igualmente por Bustelo y Ellis (2010).

En el borrador se detalla su alcance a todo tipo de organización que desee lógicamente el aseguramiento de un sistema de gestión documental así como la conformidad a sus políticas de gestión. De igual modo, se pretende demostrar mediante la "autoevaluación y autodeclaración (informe-interno) o confirmación del informe interno por terceras partes o parte externa a la organización, o certificación de su MSR por una organización externa" (2010, 1)

Considerando, dicho alcance, afirmamos que el valor de la certificación de un sistema de gestión documental es en primer lugar, el de demostrar la implantación de un sistema de gestión documental como recurso que revierte en múltiples beneficios para la organización, dado que al menos se asegura el cumplimiento de los requisitos de gestión documental y de las expectativas de los usuarios y otras partes interesadas y afectados. Un sistema de gestión documental certificado contribuye a tener un rendimiento más satisfactorio de la organización y colabora con la orientación hacia el cliente que toda organización defiende para mantenerse competitiva.

En segundo termino, cabe señalar que, mediante la certificación, se asegura la conformidad a un modelo de gestión de documentos normalizado y de reconocimiento internacional. Se trata de reflexionar sobre el prestigio de adopción de una norma que, además, es compatible con otros modelos de gestión normalizados de reconocido crédito. La certificación ubica al sistema de gestión documental en una posición de fortaleza y de visibilidad ante la organización, incidiendo en el incremento de una imagen más favorable del Archivo y del profesional en toda la organización.

En un tercer orden, se demuestra la conformidad a las políticas de gestión documental, consensuadas y aprobadas por la organización y acordes con el modelo MSR, contribuyendo de este modo a una adecuada organización y buen gobierno. En este orden, las políticas de gestión documental pueden y deben de ser coordinadas con otras políticas de gestión orientadas al cumplimiento de otros modelos de gestión. Este hecho, permite acrecentar la cohesión de modelos de gestión mediante sus estrechas interrelaciones en materia de gestión documental.

La certificación de un MSR dignifica el significado mismo de archivar, ubicándolo en idéntico plano a acciones tales como hacer calidad, alcanzar sostenibilidad, lograr seguridad, etc. 
Señalamos, en esta conjetura de valor, la importante aportación que un modelo certificado significa en la mejora de los sistemas de trabajo, puesto que la certificación asegura una adaptación continuada a los nuevos requisitos de gestión documental. De hecho, la mera implantación de la norma exige la observancia de directrices sobre la gestión, el despliegue de una arquitectura de procesos para hacerlas factibles y la elaboración de herramientas generales y específicas de gestión.

La certificación predispone al Archivo, con sus revisiones y actualizaciones periódicas, a una continua aclimatación al cambio en los contextos productivos, siendo dichas revisiones testimonios certeros de su capacidad de adaptación.

La certificación favorece la existencia de una sólida documentación demostrativa de la planificación, diseño, implementación y control del sistema sobre la que se apoyará el proceso certificador. La necesidad de documentar deriva de la necesidad de demostrar, mediante evidencias, la conformidad del sistema de gestión documental respecto a los requisitos regulados en dichas normas.

Un aspecto que debe ser altamente valorado es el potencial que supone la certificación como factor mercadotécnico. La simbología asociada a la certificación es utilizada en todas las organizaciones como un excelente activo.

La certificación repercutirá sobre las competencias y desarrollo profesional del archivero así como sobre su grado de motivación y satisfacción. El archivero se enfrenta a retos novedosos, aumentando el grado de su visibilidad en la organización. Las competencias profesionales exigidas contribuirán a predisponer al profesional hacia la innovación.

El factor certificador ha contribuido y contribuye a demostrar la diferencia entre organismos y, por tanto, puede convertirse en un activo estratégico ante determinadas decisiones o valoraciones de la Administración Pública ante gestiones, contratas, subvenciones, ayudas, etc.

Finalmente señalamos, un último valor, aunque no por ello, menos importante, el grado de compromiso y de expectación que los procesos de certificación conllevan entre todos los agentes que intervienen y, especialmente, entre los implicados. Expectativas de mejora, concienciación de los problemas y compromiso hacia el trabajo bien hecho inciden en un rendimiento mayor para el personal, los clientes y la organización.

\section{Conclusiones}

Hemos presentado el contexto certificador de los Archivos, destacando la labor certificadora asociada a modelos de administración de sistemas de gestión de la calidad. Se ha diferenciado un modelo de reconocimiento de la excelencia bastante extendido en los archivos españoles basado en la autoevaluación y fundamentado en la lógica reder, así como otras herramientas adaptadas para Administraciones públicas. Han sido expuestas las principales divergencias y sus relaciones como modelos no excluyentes. 
Se ha referenciado el compromiso de calidad que los archivos públicos españoles deben demostrar y su posibilidad de certificación, mostrando las fuentes donde el método es especificado para la obtención de las "cartas de servicios" como ejemplo de compromiso de calidad de los servicios ofrecidos en el Archivo.

Se ha resaltado la ausencia de un modelo específico de certificación de los sistemas de gestión documental y se ha analizado su proyección de futuro mediante el modelo normalizado desarrollado en el seno de ISO, en el marco de reconocimiento como un MSS, y de próxima edición, codificado como serie ISO 30300. Han sido enunciadas las principales analogías y sinergias existentes entre series MSS — sistemas normalizados de gestión-, en un intento de soslayar su correlación y compatibilidad. Se ha incidido, además, en que el modelo serie ISO 30300 no obliga a una certificación. La elección es facultativa y ofrece modelos de aproximación, mediante la autoevaluación, muy similares a los procedimientos adoptados por los modelos de reconocimiento de la excelencia, donde evaluación interna y externa o de tercera parte se confrontan hacia un informe final evaluador.

Se ha descrito el proceso certificador y su inherente proceso de acreditación que le confiere autoridad formal para la actividad de certificar. Han sido presentadas normas y agentes implicados en ambos procesos y detallada la secuencia de operaciones relativas a cada uno de ellos. Se ha enunciado el interés que el JTCG mantiene en coordinar y simplificar el proceso estudiando esta posibilidad, asegurando procedimientos similares a los acometidos para otros sistemas de gestión.

Han sido enumerados algunos de los cuantiosos valores que creemos, implica la certificación de los sistemas de gestión de documentos para una organización. Se ha acompañado cada aspecto valorado de una referencia de impacto de naturaleza cualitativa. Lógicamente, una valoración cuantitativa sólo será posible con un estudio más profundo sobre sus efectos, aunque de momento, circunscrita a la calidad. Un análisis del impacto de la certificación de sistemas de gestión documental debe esperar. Es preciso, igualmente, no desestimar ciertos inconvenientes del tipo: costes, complejidad de procedimientos, dificultades tanto en la motivación como en el reconocimiento de debilidades documentadas y su corrección (Jonquieres, 2010: 67) La certificación de sistemas de gestión de documentos esta muy próxima de ser una realidad. La serie ISO 30300, aceptada por ISO como modelo de gestión estandarizado, se muestra como lo que es: un abanico interminable de oportunidades para el archivero creativo, dotado de la capacidad de apreciar la innovación en un entorno orientado hacia la mejora continuada.

\section{Referencias}

Aenor (2010). La certificación de Aenor. El valor de la Confianza. (En línea) [consultado el 27 de octubre de 2010], de: http://www.aenor.es/aenor/certificación/procesos/proceso_certificación_aneor.asp].

Aenor: UNE-EN-ISO 19011:2002. Directrices para la auditoria de los sistemas de gestión de la calidad y/o ambiental. Madrid: autor. (En fase de revisión. Consultado borrador). 
Aenor: UNE-EN-ISO/IEC 17021:2006. Evaluación de la conformidad. Requisitos para los organismos que realizan la auditoría y la certificación de sistemas de gestión. Madrid: Autor. Revisión en inglés actualizada ISO/IEC 17021:2011 (Referenciada bajo ISO).

Aenor: UNE-EN-ISO 9000:2005. Sistemas de gestión de calidad. Fundamentos y vocabulario. Madrid: Autor.

Aenor: UNE-EN-ISO 9001:2008. Sistemas de gestión de calidad. Requisitos. Madrid: Autor.

Aenor: UNE-EN-ISO 9004:2009. Gestión para el éxito sostenido de una organización. Enfoque de gestión de la calidad. Madrid.

Aenor (2008): UNE-Gestión de documentos y evidencias electrónicas. CD-ROM, Madrid (Recopila normativa UNE-ISO 15489).

AEVAL. (2006): Guía para el desarrollo de las cartas de servicio. Madrid. Autor. [En línea) [consultado el 20 de octubre de 2010] de: http://www.aeval.es/comun/pdf/calidad/ Guia_CARTAS_2009.pdf.

AEVAL (2009): Resolución del 29 de julio del Consejo Rector de la AEVAL por el que se aprueba el procedimiento de certificación de cartas de servicios. BOE 194, 12/08/2009.

AEVAL (2009): Resolución de 18 de junio de 2009, del Consejo Rector de la Agencia Española de Evaluación de las Políticas y la Calidad de los Servicios por la que se aprueba el procedimiento de certificación de nivel de excelencia de los órganos de las Administraciones Públicas. BOE 169, 14/07/2009.

Brumm, Eugene K. (1995): Managing records for ISO 9000 compliance. Milwaukee, ASQC.

Bustelo Ruesta, C. (2009): La gestión de documentos y las evidencias en la organización. Del plano operativo al plano estratégico: una propuesta desde la normalización. Revista Española de Documentación Científica, 32 (4), 157-165.

Bustelo Ruesta, C., y Ellis, J. (2010): Better Record=Better Business. Using ISO 30300 in the strategic Management of your organization. NEN Managementseminar-Informatiehouding, La Haya, 29-10-2010. http://www.nen.nl/web/Werken/NEN-Managementseminar-Informatiehuishouding.htm.

Duff, W., y McKemmish, S. (2000): Metadata \& ISO 9000 compliance. IMJ, 34 (1).

España (2005). Real Decreto 951/2005, de 29 de julio, Por el que se establece el marco general para la mejora de la calidad en la Administración General Del Estado. (BOE, 3/09/2005).

Heredia Herrera, A. (2007): ¿Qué es el archivo? Gijón: Trea, pp. 19 y ss.

ISO (2010): ISO/DIS 30300:2010. Management system for records - Fundamentals and vocabulary. Ginebra.

ISO (2010): ISO/DIS 30301:2010. Management system for records - Requirements. Ginebra.

ISO/IEC 27006: 2007 "Information Tecnhnology - Security techniques - Requirements for bodies providing audit. And certification of information security management systems" Ginebra.

ISO (2011): ISO 17021:2011. Conformity assessment. Requirements for bodies providing audit and certification for management systems. Ginebra.

Jonquiére, M. (2010): Manual de auditoría de los sistemas de gestión. Madrid: Aenor.

Junta de Castilla y León (2006): Manual para la implantación de un sistema de gestión de calidad en un archivo. Valladolid. [En línea] (recuperado el 29 de octubre de 2010) 
de: http://www.archivoscastillayleon.jcyl.es/web/jcyl/binarios/55/998/MANUAL_CALIDAD,1.pdf?blobheader=application/pdf; charset\%3DUTF-8\&blobnocache $=$ true.

Junta de Castilla y León (2010): III Plan de intervención en el patrimonio documental de castilla y León (2010-2015). BOCYL,37, 24/02/2010. (En línea) [consultado el 29 de octubre de 2010] de: http://www.archivoscastillayleon.jcyl.es/web/jcyl/ArchivosCastillaYLeon/es/Plantilla100Detalle/1253861401355/_/1267296087729/Comunicacion.

Moro Cabero, M. (1997): El archivo de empresa. Un recurso a considerar desde la perspectiva TQM (Total Quality Management), Revista General de Información y Documentación, 7 (2), 257-275.

Moro Cabero, M. (2009): El código (ISO 15489:2001) y el fuero (normativa ISO 9000): la importancia de gestionar documentos en entornos de gestión de calidad. En Homenaje a Isabel de Torres Ramírez: Estudios de Documentación dedicada a su memoria, Universidad de Granada, 517-540.

Núñez Fernández, E. (2007): Archivos y normas ISO. Gijón:Trea.

Palom Rico, S.: ISO 9000 (1998): El proceso de certificación paso a paso. Barcelona: Granica.

Roberge, M. (2002): L'essentiel de la gestion documentaire. Québec: Gestar. 